\title{
Theory of Square-wave Voltammetry of Kinetically Controlled Two-step Electrode Reactions ${ }^{\dagger}$
}

\author{
Milivoj Lovrić* and Šebojka Komorsky-Lovrić ${ }^{*}$ \\ Department of Marine and Environmental Research, "Ruđer Bošković” Institute, 10000 Zagreb, Croatia
}

RECEIVED MAY 10, 2012; REVISED NOVEMBER 30, 2012; ACCEPTED DECEMBER 10, 2012

\begin{abstract}
An influence of electron transfer kinetics on square-wave voltammograms of two-step electrode reaction is investigated theoretically. A phenomenon of "kinetic burden" of potential inversion is described for the case of equal kinetic parameters. A linear relationship between standard rate constant and the difference between standard potentials of the second and the first charge transfers is demonstrated for the reactions with thermodynamically unstable intermediate. (doi: 10.5562/cca2126)
\end{abstract}

Keywords: square-wave voltammetry, kinetics of electrode reaction, two-step electrode reaction, intermediate, standard rate constant

\section{INTRODUCTION}

A voltammogram of reversible electrode reaction consisting of two electron transfers depends on the stability of intermediate. ${ }^{1-6}$ For electroreduction two peaks appear if the standard potential of the second electron transfer is more than hundred millivolts lower than the standard potential of the first electron transfer. ${ }^{7-10}$ Otherwise the response is a single peak. ${ }^{11-14}$ In electrochemistry of aromatic hydrocarbons the intermediate is usually stable, ${ }^{15}$ but generally the potential inversion is also possible. ${ }^{16}$ Thermodynamically unstable intermediate can be kinetically stabilized if the second charge transfer is slow. ${ }^{17}$ Furthermore, the intermediate can be stabilized by the adsorption to the electrode surface, ${ }^{18}$ or by the complexation. ${ }^{19}$ If both electron transfers are kinetically controlled, the electrode reaction appears slower if the intermediate is less stable. This phenomenon has been termed the "kinetic burden" of potential inversion. ${ }^{20}$ It is caused by the fact that the rate of electrode reaction is proportional to the concentration of intermediate. The latter is smaller if the potential inversion is higher. In this paper the manifestation of "kinetic burden" in square-wave voltammetry (SWV) is described.

\section{THE MODEL}

An electrode reaction that occurs through two consecutive steps is analyzed:

$$
\begin{aligned}
& \mathrm{Ox}^{(\mathrm{p}+2)+}+\mathrm{e}^{-} \rightleftarrows \operatorname{Int}^{(\mathrm{p}+1)+} \\
& \mathrm{Int}^{(\mathrm{p}+1)+}+\mathrm{e}^{-} \rightleftarrows \operatorname{Red}^{\mathrm{p+}}
\end{aligned}
$$

For the stationary, planar, semi-infinite diffusion, the following system of differential equations has to be solved:

$$
\begin{aligned}
& \frac{\partial c_{\mathrm{Ox}}}{\partial t}=D \frac{\partial^{2} c_{\mathrm{Ox}}}{\partial x^{2}} \\
& \frac{\partial c_{\mathrm{Int}}}{\partial t}=D \frac{\partial^{2} c_{\mathrm{Int}}}{\partial x^{2}} \\
& \frac{\partial c_{\mathrm{Red}}}{\partial t}=D \frac{\partial^{2} c_{\mathrm{Red}}}{\partial x^{2}} \\
& t=0, x \geq 0: c_{\mathrm{Ox}}=c_{\mathrm{Ox}}^{*}, c_{\mathrm{Int}}=c_{\mathrm{Red}}=0 \\
& t>0, x \rightarrow \infty: c_{\mathrm{Ox}} \rightarrow c_{\mathrm{Ox}}^{*}, c_{\mathrm{Int}} \rightarrow 0, c_{\mathrm{Red}} \rightarrow 0 \\
& x=0: D\left(\frac{\partial c_{\mathrm{Ox}}}{\partial x}\right)_{x=0}=-\frac{I_{1}}{F S}
\end{aligned}
$$

\footnotetext{
$\dagger$ This article belongs to the Special Issue devoted to the $85^{\text {th }}$ anniversary of Croatica Chemica Acta.

* Authors to whom correspondence should be addressed. (E-mail: Milivoj.Lovric@irb.hr; Sebojka.Komorsky.Lovric@irb.hr)
} 
Table 1. Meanings of symbols

\begin{tabular}{ll}
\hline$\alpha_{1}, \alpha_{2}$ & The transfer coefficients of the first and the second electron transfer steps \\
$c_{\mathrm{Ox}}, c_{\mathrm{Int}}, c_{\mathrm{Red}}$ & The concentrations of the reactant, intermediate and the product \\
$c_{\mathrm{Ox}}{ }^{*}$ & The concentration of the reactant in the bulk of solution \\
$D$ & The common diffusion coefficient \\
$\mathrm{d} E$ & The square-wave potential increment \\
$E$ & The electrode potential \\
$E_{1}^{0}, E_{2}^{0}$ & The standard potentials of the first and the second electron transfer steps \\
$E_{\mathrm{sw}}$ & The square-wave amplitude \\
$E_{\mathrm{st}}$ & The square-wave starting potential \\
$E_{\mathrm{p}}$ & The peak potential \\
$E_{\mathrm{p}, \mathrm{f}}, E_{\mathrm{p}, \mathrm{b}}$ & The peak potentials of the forward and backward components \\
$F$ & The Faraday constant \\
$f$ & The square-wave frequency \\
$\Phi$ & The dimensionless current \\
$\Delta \Phi_{\mathrm{p}}$ & The dimensionless net peak current \\
$I$ & The current \\
$\Delta I$ & The net square-wave voltammetric response \\
$I_{\mathrm{f}}, I_{\mathrm{b}}$ & The forward and backward currents \\
$\Delta I_{\mathrm{p}}$ & The net peak current \\
$k_{\mathrm{s} 1}, k_{\mathrm{s} 2}$ & The standard rate constants of the first and the second electron transfer steps \\
$\lambda_{1}, \lambda_{2}$ & The dimensionless kinetic parameters of the first and the second electrons \\
$R$ & The gas constant \\
$S$ & The electrode surface area \\
$t$ & The time \\
$x$ & The distance perpendicular to the electrode surface \\
\hline
\end{tabular}

$$
\begin{gathered}
D\left(\frac{\partial c_{\mathrm{Red}}}{\partial x}\right)_{x=0}=\frac{I_{2}}{F S} \\
\frac{I_{1}}{F S}=-k_{s, 1} \exp \left(-\alpha_{1} \varphi_{1}\right)\left[\left(c_{\mathrm{Ox}}\right)_{x=0}-\left(c_{\mathrm{Int}}\right)_{x=0} \exp \left(\varphi_{1}\right)\right] \\
\frac{I_{2}}{F S}=-k_{s, 2} \exp \left(-\alpha_{2} \varphi_{2}\right)\left[\left(c_{\mathrm{Int}}\right)_{x=0}-\left(c_{\mathrm{Red}}\right)_{x=0} \exp \left(\varphi_{2}\right)\right] \\
\varphi_{1}=\frac{F}{R T}\left(E-E_{1}^{0}\right) \\
\varphi_{2}=\frac{F}{R T}\left(E-E_{2}^{0}\right)
\end{gathered}
$$

The meanings of all symbols are reported in the Table 1 . Differential equations (3)-(5) are solved by Laplace transformations and by the method of numerical integration proposed by Olmstead and Nicholson. ${ }^{21}$ The solution is the system of recursive formulae for the dimensionless current $\Phi_{i}=I_{i}\left(F S c_{O x}^{*}\right)^{-1}(D f)^{-1 / 2}$, where $i=1$ or 2. The sum $\Phi=\Phi_{1}+\Phi_{2}$ is reported as a function of electrode potential.

$$
\begin{aligned}
& \Phi_{1, m}= \\
& Z_{m}\left[\frac{-5 \sqrt{\pi}}{\exp \left(\varphi_{1, m}\right) \sqrt{2}}+V_{m} \sum_{j=1}^{m-1} \Phi_{1, j} s_{m-j+1}+W_{m} \sum_{j=1}^{m-1} \Phi_{2, j} s_{m-j+1}\right] \\
& \Phi_{2, m}=\Phi_{1, m}\left[\frac{5 \sqrt{\pi} \exp \left(-\left(1-\alpha_{1}\right) \varphi_{1, m}\right)}{\lambda_{1} \sqrt{2}}+\left(1+\exp \left(-\varphi_{1, m}\right)\right)\right] \\
& +\frac{5 \sqrt{\pi}}{\exp \left(\varphi_{1, m}\right) \sqrt{2}}-\frac{5 \sqrt{\pi}}{\sqrt{2}}+ \\
& \left(1+\exp \left(\varphi_{1, m}\right)\right) \exp \left(-\varphi_{1, m}\right) \sum_{j=1}^{m-1} \Phi_{1, j} s_{m-j+1}-\sum_{j=1}^{m-1} \Phi_{2, j} s_{m-j+1} \\
& Z_{m}=N_{m}^{-1} \lambda_{1} \exp \left(\left(1-\alpha_{1}\right) \varphi_{1, m}\right) \sqrt{2} . \\
& \cdot\left[5 \sqrt{\pi}+\lambda_{2} \exp \left(-\alpha_{2} \varphi_{2, m}\right)\left(1+\exp \left(\varphi_{2, m}\right)\right) \sqrt{2}\right] \\
& N_{m}=\left[5 \sqrt{\pi}+\lambda_{1} \exp \left(-\alpha_{1} \varphi_{1, m}\right)\left(1+\exp \left(\varphi_{1, m}\right)\right) \sqrt{2}\right] \\
& \cdot\left[5 \sqrt{\pi}+\lambda_{2} \exp \left(-\alpha_{2} \varphi_{2, m}\right)\left(1+\exp \left(\varphi_{2, m}\right)\right) \sqrt{2}\right] \\
& -2 \lambda_{1} \lambda_{2} \exp \left(\left(1-\alpha_{1}\right) \varphi_{1, m}\right) \exp \left(-\alpha_{2} \varphi_{2, m}\right)
\end{aligned}
$$


Table 2. The SWV dimensionless net peak currents and peak potentials of fast and reversible two-step electrode reactions for $E_{\mathrm{SW}}$ $=50 \mathrm{mV}$ and $\mathrm{d} E=-5 \mathrm{mV}$

\begin{tabular}{|c|c|c|c|c|c|c|}
\hline$\frac{E_{2}^{0}-E_{1}^{0}}{\mathrm{~V}}$ & $\frac{E_{s t}-E_{1}^{0}}{\mathrm{~V}}$ & $-\Delta \Phi_{p, 1}$ & $\frac{E_{p, 1}-E_{1}^{0}}{\mathrm{~V}}$ & $-\Delta \Phi_{p, 2}$ & $\frac{E_{p, 2}-E_{1}^{0}}{\mathrm{~V}}$ & $\frac{E_{\min }-E_{1}^{0}}{\mathrm{~V}}$ \\
\hline 0.200 & 0.400 & 1.9063 & 0.100 & - & - & - \\
\hline 0.100 & 0.350 & 1.8752 & 0.050 & - & - & - \\
\hline 0.050 & 0.350 & 1.8184 & 0.025 & - & - & - \\
\hline 0.000 & 0.300 & 1.6835 & 0.000 & - & - & - \\
\hline-0.050 & 0.300 & 1.4073 & -0.025 & - & - & - \\
\hline-0.100 & 0.250 & 0.9819 & -0.050 & - & - & - \\
\hline-0.111 & 0.250 & 0.8805 & -0.035 & 0.8834 & -0.080 & -0.055 \\
\hline-0.120 & 0.250 & 0.8316 & -0.015 & 0.8349 & -0.105 & -0.060 \\
\hline-0.130 & 0.250 & 0.8022 & -0.010 & 0.8049 & -0.120 & -0.065 \\
\hline-0.150 & 0.250 & 0.7734 & -0.005 & 0.7751 & -0.145 & -0.075 \\
\hline-0.175 & 0.250 & 0.7596 & 0.000 & 0.7609 & -0.175 & -0.090 \\
\hline-0.200 & 0.250 & 0.7549 & 0.000 & 0.7558 & -0.200 & -0.100 \\
\hline
\end{tabular}

$V_{m}=\left[\begin{array}{l}-\left(1+\exp \left(\varphi_{1, m}\right)\right) 5 \sqrt{\pi}-\lambda_{2} \sqrt{2} \exp \left(-\alpha_{2} \varphi_{2, m}\right) \\ -\lambda_{2} \sqrt{2}\left(1+\exp \left(\varphi_{1, m}\right)\right) \exp \left(\left(1-\alpha_{2}\right) \varphi_{2, m}\right)\end{array}\right]$
$\left[5 \sqrt{\pi}+\lambda_{2} \sqrt{2} \exp \left(-\alpha_{2} \varphi_{2, m}\right)\left(1+\exp \left(\varphi_{2, m}\right)\right)\right]^{-1} \exp \left(-\varphi_{1, m}\right)$

$W_{m}=5 \sqrt{\pi}\left[5 \sqrt{\pi}+\lambda_{2} \sqrt{2} \exp \left(-\alpha_{2} \varphi_{2, m}\right)\left(1+\exp \left(\varphi_{2, m}\right)\right)\right]^{-1}$

$\lambda_{i}=k_{s, i}(D f)^{-1 / 2} \quad(i=1,2)$

$S_{k}=\sqrt{k}-\sqrt{k-1}$

$1 \leq m \leq M$

$M=50\left(E_{\text {start }}-E_{\text {final }}\right) / \mathrm{d} E$

\section{RESULTS AND DISCUSSION}

Square-wave voltammogram of fast and reversible twostep electrode reaction depends on standard potentials of individual electron transfers and on the parameters of $\mathrm{SWV}$ excitation signal. If $E_{2}^{0}-E_{1}^{0}>-0.110 \mathrm{~V}$, the response is a single peak appearing at the medium potential $\left(E_{1}^{0}+E_{2}^{0}\right) / 2$. Dimensionless net peak currents $-\Delta \Phi_{p}=-\Delta I_{p}\left(F S c_{\mathrm{Ox}}^{*}\right)^{-1}(D f)^{-1 / 2}$ are reported in Table 2, for $E_{\mathrm{SW}}=50 \mathrm{mV}$ and $\mathrm{d} E=-5 \mathrm{mV}$. They are diminished proportionally to the decreasing of the difference $E_{2}^{0}-E_{1}^{0}$. The voltammograms are split into two peaks if $E_{2}^{0}-E_{1}^{0} \leq-0.111 \mathrm{~V}$. The potential of minimum between two maxima of the split response is equal to the medium potential. The potentials of two peaks are equal to the corresponding standard potentials if $E_{2}^{0}-E_{1}^{0}<-0.150 \mathrm{~V}$.
The voltammograms of kinetically controlled electrode reactions are generally smaller than the responses (19) of reversible reactions and depend on the dimensionless kinetic parameters $\lambda_{i}=k_{s, i}(D f)^{-1 / 2}$, where $i=1$ or $2 .{ }^{17}$ Figure 1 shows SWV responses of two quasireversible two-step electrode reactions, which have equal kinetic parameters but different standard potentials of the se20) cond electron transfer. If $E_{2}^{0}=E_{1}^{0}$, the net peak current equals 1.621 and the peak potential is $-0.005 \mathrm{~V} v s . E_{1}^{0}$. These values are similar to those reported in Table 2, for reversible electrode reaction. The extremes of components are the following: $\Phi_{\mathrm{p}, \mathrm{f}}=-1.0464, E_{\mathrm{p}, \mathrm{f}}-E_{1}^{0}=$ $0.005 \mathrm{~V}, \Phi_{\mathrm{p}, \mathrm{b}}=0.5837$ and $E_{\mathrm{p}, \mathrm{b}}-E_{1}^{0}=-0.010 \mathrm{~V}$. However, if $E_{2}^{0}-E_{1}^{0}=0.4 \mathrm{~V}$ and the kinetic parameters are the same, the net peak current decreases to 0.4405 and the peak potential is $0.130 \mathrm{~V}$ vs. $E_{1}^{0}$, which is 70 $\mathrm{mV}$ lower than the medium potential $\left(E_{1}^{0}+E_{2}^{0}\right) / 2$. Furthermore, the components are also diminished: $\Phi_{\mathrm{p}, \mathrm{f}}=-0.5038, E_{\mathrm{p}, \mathrm{f}}-E_{1}^{0}=0.115 \mathrm{~V}, \Phi_{\mathrm{p}, \mathrm{b}}=0.0251$ and $E_{\mathrm{p}, \mathrm{b}}-E_{1}^{0}=0.205 \mathrm{~V}$. All these are characteristics of the response of irreversible electrode reaction. ${ }^{22}$ Hence, Figure 1 is an example of "kinetic burden" of potential inversion in SWV.

Under equilibrium conditions, the stability of intermediate is the best estimated at the medium potential: ${ }^{17}$

$$
c_{\text {Int,eq }, \bar{E}}=c_{\text {tot }}\left[1+2 \exp \left(F\left(E_{2}^{0}-E_{1}^{0}\right) / 2 R T\right)\right]^{-1}
$$

In this equation $c_{\text {tot }}$ is the sum of concentrations of the reactant, intermediate and product. If $E_{2}^{0}=E_{1}^{0}$, $c_{\text {Int }, \mathrm{eq}, \bar{E}} / c_{\text {tot }}=0.333$ and if $E_{2}^{0}-E_{1}^{0}=0.4 \mathrm{~V}, c_{\text {Int }, \mathrm{eq}, \bar{E}} / c_{\text {tot }}$ $=2.08 \times 10^{-4}$. So, the stability of intermediate decreases 1600 times if the difference $E_{2}^{0}-E_{1}^{0}$ increases from zero to $0.4 \mathrm{~V}$. This is one of reasons for the difference between the voltammograms in Figures 1A and 1B. 

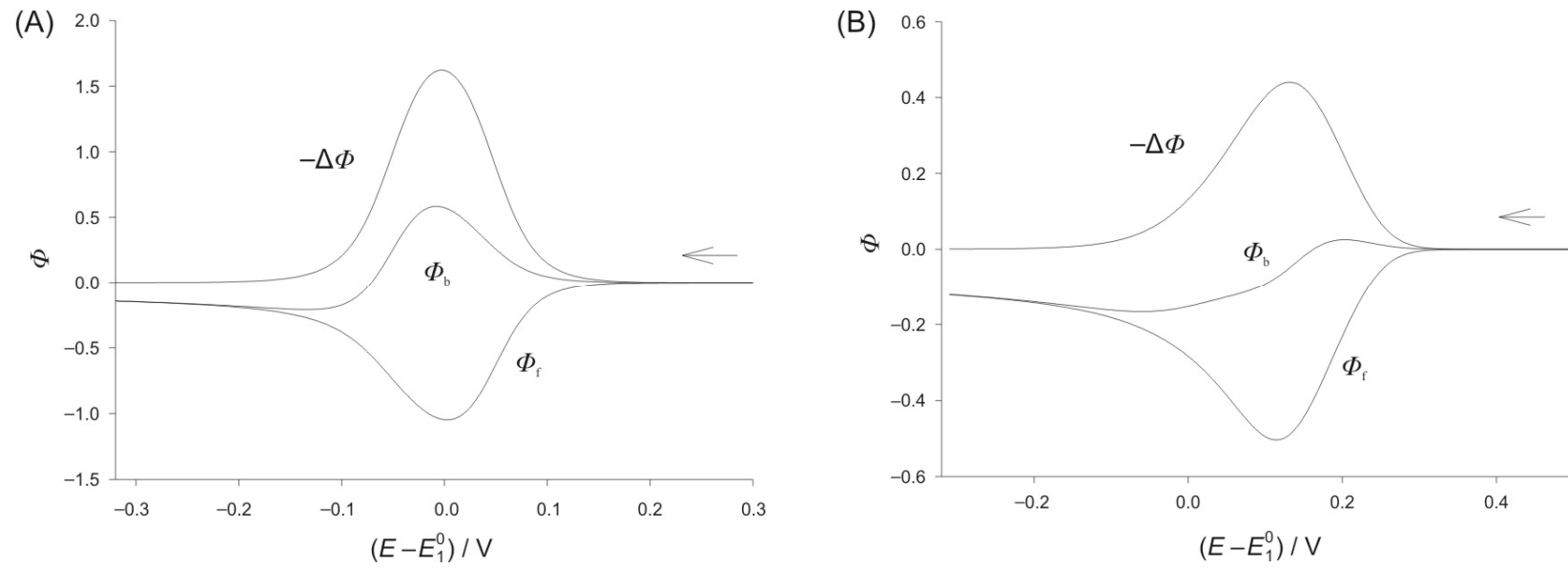

Figure 1. Theoretical square-wave voltammograms of two-step electrode reaction (1) and (2); $\lambda_{1}=3, \lambda_{2}=3, \alpha_{1}=0.5, \alpha_{2}=0.5$, $E_{\mathrm{SW}}=50 \mathrm{mV}, d E=-5 \mathrm{mV}$ and $\left(E_{2}^{0}-E_{1}^{0}\right) / \mathrm{V}=0(\mathrm{~A})$ and $0.4(\mathrm{~B})$. A dimensionless net current $(-\Delta \Phi)$ and its forward $\left(\Phi_{\mathrm{f}}\right)$ and backward $\left(\Phi_{\mathrm{b}}\right)$ components are shown.

The dependences of net peak currents and peak potentials on the logarithm of kinetic parameter $\lambda_{1}$ are shown in Figures 2 and 3, for $E_{2}^{0}=E_{1}^{0}$ and $E_{2}^{0}-E_{1}^{0}=$ $0.4 \mathrm{~V}$, respectively. They are characterized by two critical parameters $\left(\lambda_{1}\right)_{1 / 2}$ and $\left(\lambda_{1}\right)_{\text {cross }}$ which depend on the difference $E_{2}^{0}-E_{1}^{0}$. All peak currents depend on $\log \lambda_{1}$ sigmoidally. In Figure 2 the net peak current reaches the half of limiting value for $\log \left(\lambda_{1}\right)_{1 / 2}=-0.27$, which is marked by the small vertical line above the abscissa. In the same figure the net peak potentials and the peak potentials of forward component depend linearly on $\log \lambda_{1}$, with the common slope $\Delta E_{\mathrm{p}, \mathrm{f}} / \Delta \log \lambda_{1}=0.118 \mathrm{~V}$. The peak potentials of backward component are also linear function of $\log \lambda_{1}$, with the slope $-0.107 \mathrm{~V}$. These relationships are approximated by the straight lines 1 and 2. The intersection of these lines is defined by $\log \left(\lambda_{1}\right)_{\text {cross }}=-0.519$, which is also marked. For some

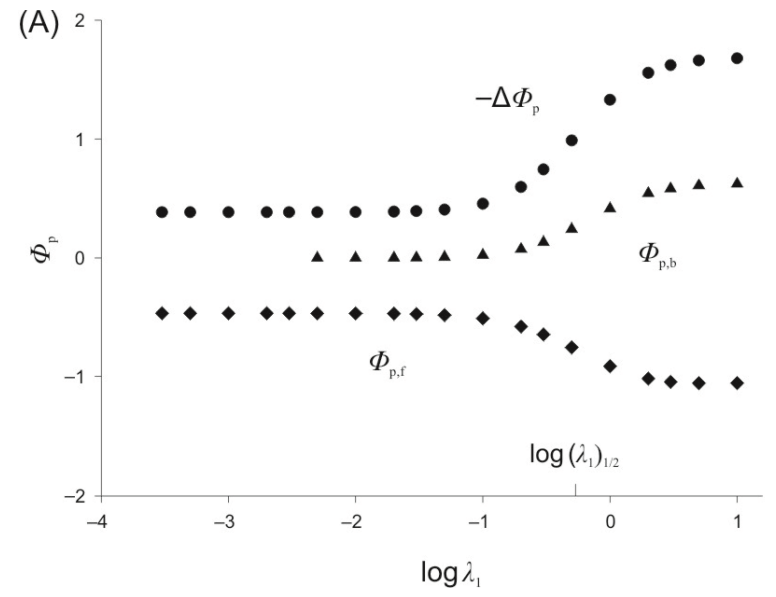

quasireversible reactions, these critical parameters can be measured by the variation of frequency. Considering that $\alpha_{1}=0.5$ and $\alpha_{2}=0.5$, Figure 2B shows that for equal kinetic parameters $\left(\lambda_{1}=\lambda_{2}\right)$ the slope $\Delta E_{\mathrm{p}, \mathrm{f}} /$ $\Delta \log f=-2.3 R T / 2 \alpha F$ suggests that a single electron is transferred. This confirms earlier findings that the response of some two-step electrode reactions may resemble the response of one-electron reaction. ${ }^{3,8,11}$ This phenomenon is a consequence of equal kinetic parameters $\lambda_{1}$ and $\lambda_{2}$, and not of equal standard potentials $E_{1}^{0}$ and $E_{2}^{0}$, which can be concluded from the fact that for $E_{2}^{0}-E_{1}^{0}=0.4 \mathrm{~V}$ the slope of the straight line 1 is also $0.118 \mathrm{~V}$ (see Figure 3B). Moreover, this slope is independent of standard potentials within the interval $-0.2 \leq$ $\left(E_{2}^{0}-E_{1}^{0}\right) / \mathrm{V} \leq 0.6$. It is different with the slope $\Delta E_{\mathrm{p}, \mathrm{b}}$ / $\Delta \log \lambda_{1}$ which is equal to $-0.029 \mathrm{~V}$ in Figure $3 \mathrm{~B}$ and $-0.030 \pm 0.005 \mathrm{~V}$ within the interval $0.2 \leq\left(E_{2}^{0}-E_{1}^{0}\right) /$

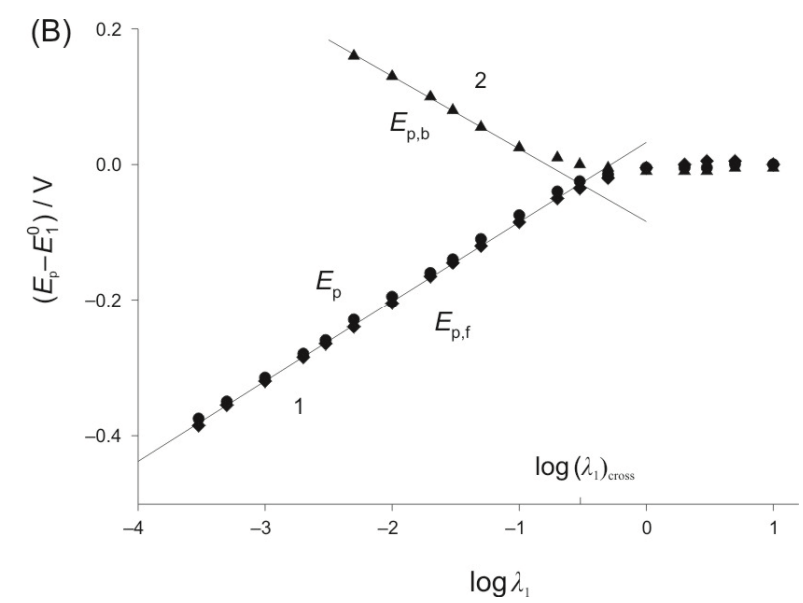

Figure 2. Dependence of dimensionless net peak current and peak currents of forward and backward components (A) and net peak potential and the potentials of extremes of components (B) on the logarithm of kinetic parameter $\lambda_{1} \cdot E_{2}^{0}=E_{1}^{0}, \lambda_{2}=\lambda_{1}$ and all other parameters are as in Figure 1. The straight lines 1 and 2 are linear approximations. 

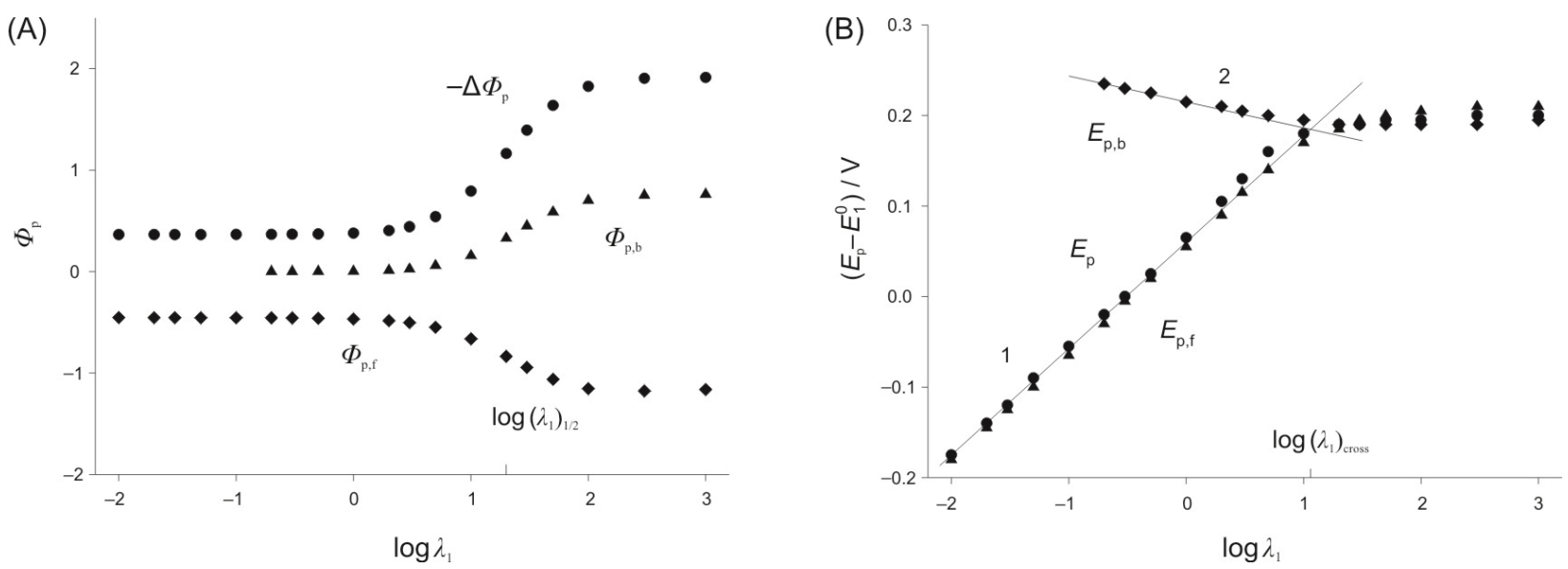

Figure 3. Dependence of dimensionless net peak current and peak currents of forward and backward components (A) and net peak potential and the potentials of extremes of components (B) on the logarithm of kinetic parameter $\lambda_{1} . E_{2}^{0}-E_{1}^{0}=0.4 \mathrm{~V}$, $\lambda_{2}=\lambda_{1}$ and all other parameters are as in Figure 1.

$\mathrm{V} \leq 0.6$. For the difference $E_{2}^{0}-E_{1}^{0}=0.4 \mathrm{~V}$ the critical parameters are bigger than in Figure 2: $\log \left(\lambda_{1}\right)_{1 / 2}=1.30$ and $\log \left(\lambda_{1}\right)_{\text {cross }}=1.06$. The dependence of these critical parameters on the difference between standard potentials is shown in Figure 4. These functions are defined as in Figures 2 and 3. For $E_{2}^{0}-E_{1}^{0}>0$ these relationships can be approximated by the following equations: $\log \left(\lambda_{1}\right)_{1 / 2}=4.28 \times\left(E_{2}^{0}-E_{1}^{0}\right)-0.45$ and $\log \left(\lambda_{1}\right)_{\text {cross }}=$ $4.15 \times\left(E_{2}^{0}-E_{1}^{0}\right)-0.62$. Physical meaning of these equations is that it is not possible to determine the standard rate constants of two-step electrode reaction with unstable intermediate by the variation of squarewave frequency. However, they show the relationship between the rate constant, the difference $E_{2}^{0}-E_{1}^{0}$ and the critical frequency. If it is possible to measure linear relationships between $E_{\mathrm{p}, \mathrm{f}}, E_{\mathrm{p}, \mathrm{b}}$ and the logarithm of frequency, one can determine the logarithm of critical frequency $\log f_{\text {cross }}$ at which these lines intersect. This frequency is related to the standard rate constant as follows:

$$
\log k_{s, 1}=\frac{1}{2} \log \left(f_{\text {cross }}\right)+4.15 \times\left(E_{2}^{0}-E_{1}^{0}\right)-0.62+\frac{1}{2} \log D(26)
$$

Equation (26) applies under the conditions used in this work, namely that both electron transfers are equally fast $\left(\lambda_{2}=\lambda_{1}\right)$, that $\alpha_{1}=\alpha_{2}=0.5$ and that $E_{\mathrm{SW}}=50$ $\mathrm{mV}$ and $\mathrm{d} E=-5 \mathrm{mV}$. The first condition is most probably fulfilled if a single SWV net peak appears at all frequencies. This is because the SWV response of electrode reactions with thermodynamically unstable intermediate may split into two peaks at highest frequencies if the second electron transfer is much slower than the first one. ${ }^{17}$ If the first charge transfer is slower than the
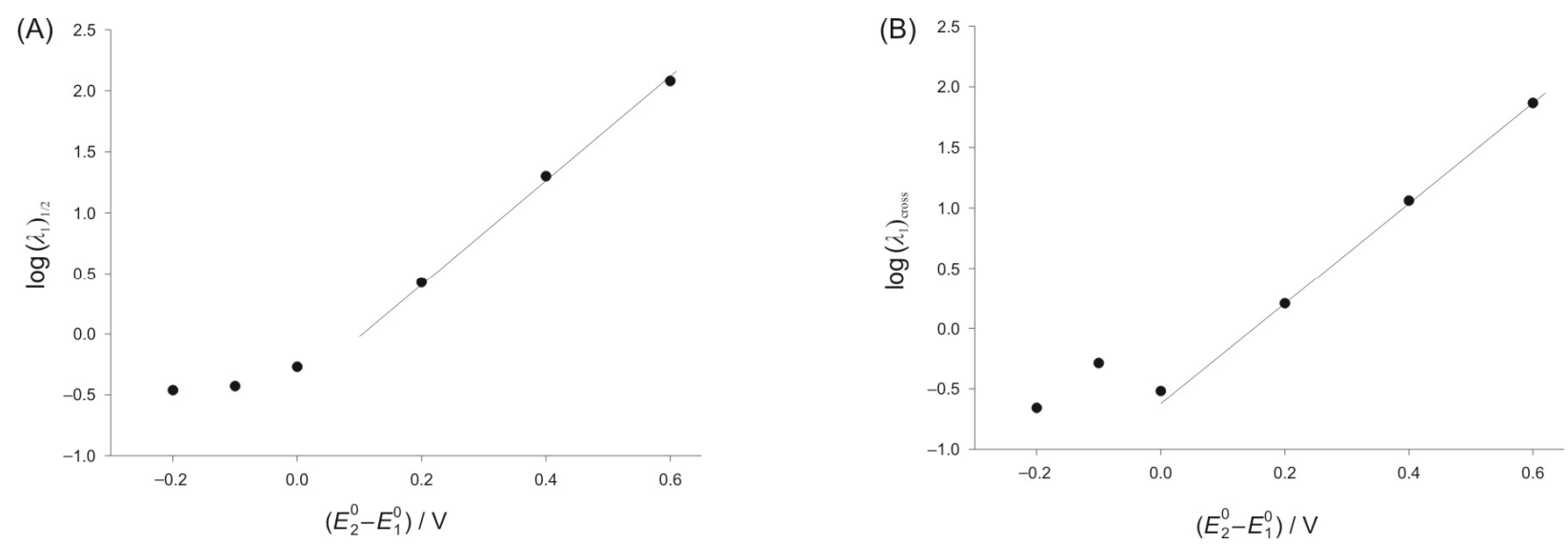

Figure 4. Dependence of critical parameters determining the half-height of net peak currents (A) and the intercept of linear dependences of $E_{p, f}$ and $E_{p, b}$ on $\log \lambda_{1}(\mathrm{~B})$ on the difference between standard potentials of the second and the first charge transfers. All parameters are as in Figure 1. 

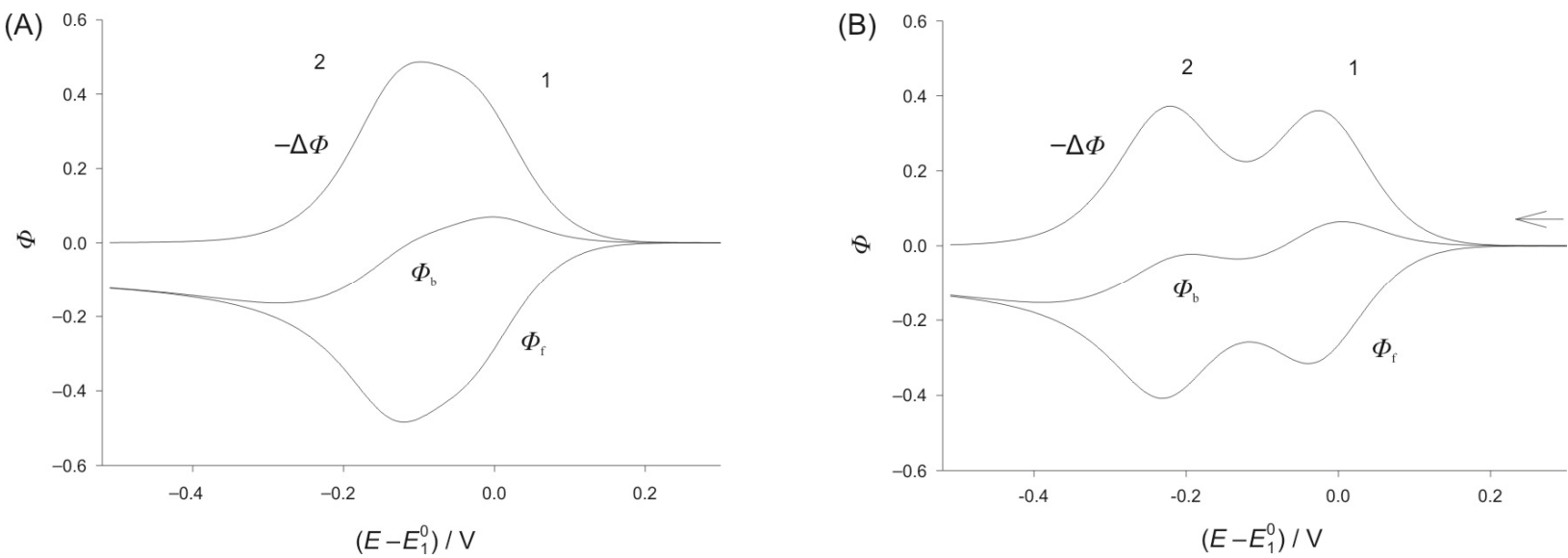

Figure 5. Theoretical square-wave voltammograms of two-step electrode reaction (1) and (2); $\lambda_{1}=\lambda_{2}=0.2,\left(E_{2}^{0}-E_{1}^{0}\right) / \mathrm{V}=$ -0.1 (A) and -0.2 (B) and all other parameters are as in Figure 1.

second one, the response may also split in two peaks because of the influence of the backward component. ${ }^{23}$

Figure 4 shows that critical parameters of two-step electrode reaction with stable intermediate deviate from eq. (26). This is because of the separation of steps, as can be seen in Figures 5 and 6. In the first phase of this process, for $E_{2}^{0}-E_{1}^{0}=-0.1 \mathrm{~V}$, the backward component of the first electron transfer is separated from the forward component of the second charge transfer (see Figure 5A). The net response is a single peak appearing at the potential which is close to the potential of minimum of forward component. Minimum separation between peak potentials of components is $E_{\mathrm{p}, \mathrm{b}, 1}-E_{\mathrm{p}, \mathrm{f}, 2}=$ $55 \mathrm{mV}$. This is shown in Figure 6A. Note that in Figure $2 \mathrm{~B}$ this separation is only $5 \mathrm{mV}$. The slope of line 2 in Figure $6 \mathrm{~A}$ is $-0.113 \mathrm{~V}$. If $E_{2}^{0}-E_{1}^{0}=-0.2 \mathrm{~V}$ the two steps are fully separated and the response consists of two peaks (see Figure 5B). The relationships between all peak potentials and the logarithm of kinetic parame-

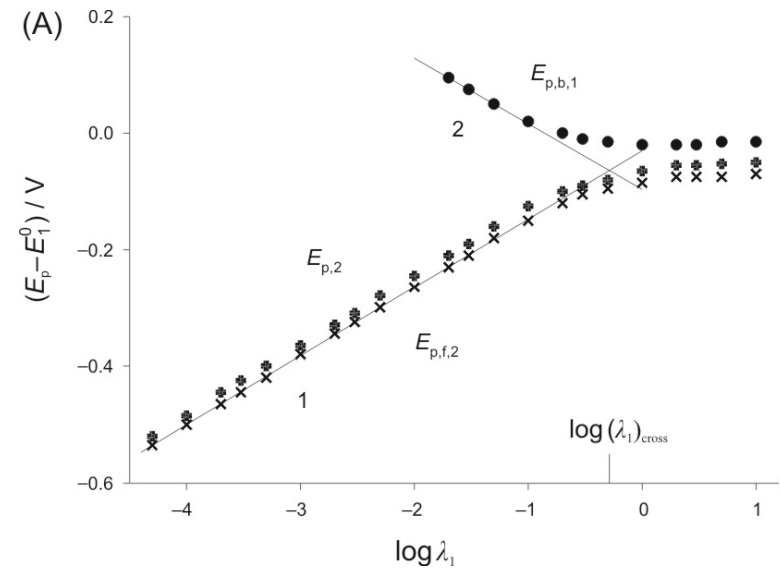

ter $\lambda_{1}$ are shown in Figure 6B. As $\lambda_{2}=\lambda_{1}$ and $\alpha_{2}=\alpha_{1}$, the straight lines 1 and 3 are parallel. The slope of the line 2 is $-0.126 \mathrm{~V}$. This figure shows that the influence of kinetics on two separated steps of electrode reaction with stable intermediate can be analyzed independently. The transfer coefficients $\alpha_{1}$ and $\alpha_{2}$ can be determined from the dependence of the first and the second net peak potentials on the logarithm of frequency, and the standard rate constant of the first electron transfer can be estimated from the critical kinetic parameter: $\log k_{s, 1}=\frac{1}{2} \log f_{\text {cross }}-0.66+\frac{1}{2} \log D$, which applies for the conditions reported in Figure 6.

\section{CONCLUSIONS}

In this paper we described square-wave voltammograms of a particular type of two-step electrode reactions in which the charge transfers were equally fast and sym-

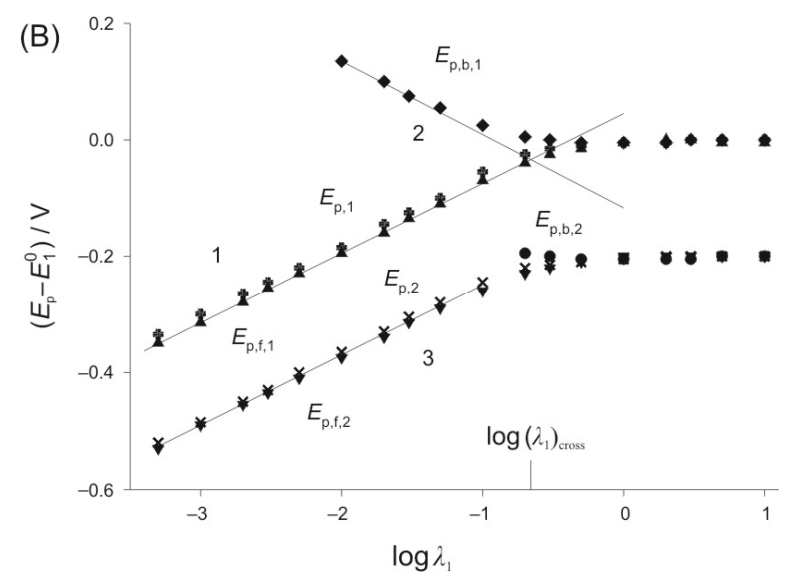

Figure 6. Dependence of net peak potential and the potentials of extremes of components on the logarithm of kinetic parameter $\lambda_{1} .\left(E_{2}^{0}-E_{1}^{0}\right) / \mathrm{V}=-0.1(\mathrm{~A})$ and $-0.2(\mathrm{~B}), \lambda_{2}=\lambda_{1}$ and all other parameters are as in Figure 1. 
metrical. The apparent stability of intermediate of such reactions can not be changed by the variation of frequency. In the case of unstable intermediate, the apparent reversibility of electrode reaction depends on the difference between standard potentials of the second and the first charge transfers. This means that the critical kinetic parameters, which determine ideal transitions from reversible to irreversible reactions, depend on the difference $E_{2}^{0}-E_{1}^{0}$. The consequence is that the standard rate constants can not be estimated by the variation of frequency. However, our calculation shows that there is a linear relationship between the logarithm of standard rate constant and the difference in standard potentials, which is defined by equation (26).

\section{REFERENCES}

1. D. S. Polcyn and I. Shain, Anal. Chem. 38 (1966) 370-375.

2. F. Ammar and J. M. Saveant, J. Electroanal. Chem. 47 (1973) 215-221.

3. I. Ružić, J. Electroanal. Chem. 52 (1974) 331-354.

4. J. Galvez, R. Saura, A. Molina, and T. Fuente, J. Electroanal. Chem. 139 (1982) 15-36.

5. M. W. Lehmann and D. H. Evans, Anal. Chem. 71 (1999) 1947-1950.

6. S. R. Belding, J. G. Limon-Petersen, E. J. F. Dickinson, and R. G. Compton, Angew. Chem. Int. Ed. 49 (2010) 9242-9245.
7. V. Mirčeski and R. Gulaboski, Croat. Chem. Acta 76 (2003) 37-48.

8. A. Molina, M. M. Moreno, C. Serna, M. Lopez-Tenes, J. Gonzalez, and N. Abenza, J. Phys. Chem. C 111 (2007) 12446-12453.

9. R. Gulaboski, J. Solid State Electrochem. 13 (2009) 1015-1024.

10. R. Gulaboski and Lj. Mihajlov, Biophys. Chem. 155 (2011) $1-9$.

11. A. Molina, C. Serna, M. Lopez-Tenes, and M. M. Moreno, J. Electroanal. Chem. 576 (2005) 9-19.

12. M. Lopez-Tenes, A. Molina, C. Serna, M. M. Moreno, and J. Gonzalez, J. Electroanal. Chem. 603 (2007) 249-259.

13. S. R. Belding, R. Baron, E. J. F. Dickinson, and R. G. Compton, J. Phys. Chem. C 113 (2009) 16042-16050.

14. O. V. Klymenko, I. Svir, and C. Amatore, Electrochem. Commun. 12 (2010) 1378-1382.

15. D. H. Evans, Chem. Rev. 108 (2008) 2113-2144.

16. D. H. Evans and M. W. Lehmann, Acta Chem. Scand. 53 (1999) 765-774.

17. Š. Komorsky-Lovrić and M. Lovrić, J. Electroanal. Chem. 660 (2011) 22-25.

18. M. Lovrić and Š. Komorsky-Lovrić, Int. J. Electrochem. (2012) ID 596268.

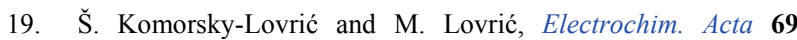
(2012) 60-64.

20. D. H. Evans, Acta Chem. Scand. 52 (1998) 194-197.

21. M. L. Olmstead and R. S. Nicholon, J. Electroanal. Chem. 16 (1968) 145-151.

22. V. Mirčeski, Š. Komorsky-Lovrić, and M. Lovrić, Square-Wave Voltammetry, Springer, Berlin, 2007, pp. 17.

23. Š. Komorsky-Lovrić and M. Lovrić, Electrochim. Acta 56 (2011) 7189-7193. 